\title{
Influencia de los Centros Escolares sobre el Rendimiento Académico en Latinoamérica
}

\author{
Pamela Woitschach', Rubén Fernández-Alonso ${ }^{2 \star}$, Rosario Martínez-Arias ${ }^{1}$ y José Muñiz ${ }^{3}$ \\ Universidad Complutense de Madrid \\ ${ }^{2}$ Consejería de Educación y Cultura del Principado de Asturias y Universidad de Oviedo \\ 3Universidad de Oviedo
}

\begin{abstract}
Resumen. La estimación del efecto de los centros es la piedra angular en el estudio de la eficacia escolar. En Latinoamérica, las conclusiones sobre el efecto de los centros no siempre son consistentes. Esta investigación analiza la influencia del contexto educativo de los centros sobre el rendimiento académico. La muestra es de 63.750 estudiantes, de 2.955 escuelas de quince países latinoamericanos. La edad media del alumnado es de 12,4 años (DT =0,94). El 69,4\% asiste a un centro público, el $50,3 \%$ son mujeres y el $20,4 \%$ ha repetido al menos un curso. Se emplea el análisis jerárquicolineal de dos y tres niveles, las variables de ajuste utilizadas son los antecedentes demográficos y socioeconómicos del alumno, y titularidad, ruralidad, infraestructura y nivel socioeconómico de los centros. Los resultados muestran que el efecto bruto de los centros es del 33\% para Ciencias Naturales y $28 \%$ para Matemáticas y Lectura. El impacto de las variables socioeconómicas reduce esta variabilidad entre en $35 \%$ y el $68 \%$. El efecto neto de los centros fue de $25 \%$ en Ciencias Naturales, $23 \%$ en Matemática y $13 \%$ en Lectura. Se discuten estos resultados y se analizan posibles implicaciones para las políticas educativas en Latinoamérica.
\end{abstract}

Palabras clave: Latinoamérica, equidad, educación primaria, rendimiento académico, modelos jerárquicos lineales.

\section{Influence of the Schools on Academic Performance in Latin America}

Abstract. Estimating the effect of schools is the cornerstone in the study of school effectiveness. In Latin America, the conclusions about the effect of the schools are not always consistent. This research analyzes the influence of background variables on the academic performance. The sample is of 63,750 students, of 2,955 schools of fifteen Latin American countries. The average age of students is 12.4 years $(S D=0.94) .69 .4 \%$ attend a public school, $50.3 \%$ are women and $20.4 \%$ have repeated at least one course. It uses the hierarchical-linear analysis of two and three levels, the adjustment variables used are the demographic and socio-economic background of the student, and private school, rural school, infrastructure and socio-economic level of the schools. The results show that the gross effect of the centers is 33\% for Sciences and 28\% for Mathematics and Reading. The impact of socioeconomic variables reduces this variability by $35 \%$ to $68 \%$. The net effect of the centers was $25 \%$ in Sciences, $23 \%$ in Mathematics and $13 \%$ in Reading. We discuss these results and analyze possible implications for educational policies in Latin America.

Keywords: Latin America, equity, primary education, academic achievement, linear hierarchical models.

La iniciativa Educación Para Todos de la Organización de las Naciones Unidas para la Educación, la Ciencia y la Cultura (UNESCO, 2000) persigue entre sus objetivos mitigar la

Recibido: 30/05/2017 - Aceptado: 22/06/2017 - Avance online: 28/06/2017

*Correspondencia: Rubén Fernández Alonso.

Consejería de Educación y Cultura del Principado de Asturias y

Universidad de Oviedo.

C.P: 33007, Oviedo, España.

E-mail: fernandezaruben@uniovi.es

Woitschach, P., Fernández, R., Martínez, R. \& Muñiz, J. (2017). Influencia de los Centros Escolares sobre el Rendimiento Académico en Latinoamérica. Revista de Psicología y Educación, 12(2), 138-154, https://doi.

org/10.23923/rpye2017.12.152 desigualdad en materia de educación y suprimir las discriminaciones en las posibilidades de aprendizaje de grupos desfavorecidos. La investigación sobre la eficacia escolar aborda esta problemática, contribuyendo con un amplio volumen de hallazgos (Scheerens, 2016; Scheerens, Witziers, y Steen, 2013; Teddlie y Reynolds, 2000; Towsend, 2007). De acuerdo con Teddlie y Reynolds (2000)

1699-9517/@ 2017 Asociación Científica de Psicología y Educación (ACIPE). Publicado por Consejo General de Colegios Oficiales de Psicólogos, España. Este es un artículo Open Access bajo la CC BY-NC-ND licencia (http://creativecommons.org/licencias/by-nc-nd/4.0/). 
los estudios sobre eficacia escolar tienen dos grandes propósitos, por un lado estimar los efectos de la escuela, ya sean brutos o bajo alguna definición de eficacia relativa, es decir, una vez eliminado el influjo de las variables de contexto 0 antecedentes al proceso educativo, y por otro, analizar los factores asociados, es decir, los procesos y características que permiten que las escuelas promuevan el aprendizaje del alumnado más allá de lo que sería esperable en función de las variables de contexto. El presente trabajo se encuadra dentro de la primera finalidad, y pretende conocer el efecto del centro escolar entendido como la proporción de varianza de los resultados educativos que puede ser imputada a las variables de proceso educativo de los centros estimada, bien como las diferencias brutas entre los centros, bien como la varianza por explicar una vez descontadas las variables que describen el contexto escolar en América Latina (Murillo, 2005).

El estudio del efecto bruto del centro educativo, que operativamente se define como el porcentaje de las diferencias imputadas al factor "centro escolar" en un ANOVA de efectos aleatorios o como la correlación intraclase de un modelo multinivel sin predictores, es la línea primigenia y más antigua de la eficacia escolar, y cuenta con una tradición de cinco décadas (Murillo, 2005). En América Latina la investigación sobre los efectos escolares se inició hace dos décadas, por lo que en la actualidad ya se dispone de un amplio conjunto de trabajos (Blanco-Bosco, 2009; Murillo, 2003, 2007, 2008). Sin duda, los estudios latinoamericanos con mayor alcance son los realizados por el Laboratorio Latinoamericano de Evaluación de la Calidad de la Educación (LLECE), que, hasta la fecha, ha realizado tres ediciones del Estudio Regional Comparativo y Explicativo: la primera (denominada PERCE) en 1997, la segunda (SERCE) en 2006 y la tercera (TERCE) en 2013. Se trata de una evaluación internacional, donde han participado una veintena de países de la región y cuya finalidad es analizar los logros del aprendizaje del alumnado y los factores asociados a este logro (UNESCO-OREALC y LLECE, 2000, 2010, 2016b).
Sin embargo, pese al corpus de investigación acumulado (o precisamente por ello) las conclusiones sobre el efecto bruto del centro no siempre son consistentes, llegando incluso a parecer contradictorias. Por ejemplo, los análisis secundarios realizados con datos de las comparaciones internacionales de la región generalmente informan de efectos brutos similares a los publicados en el estudio original (Miralles, Castejón, Pérez, y Gilar, 2012). Sin embargo, no faltan ocasiones donde los autores comunican efectos superiores (CastroAristizabal, Castillo-Caicedo, y MendozaParra, 2016; Doneschi, 2017) o inferiores (Cervini, 2012; Murillo y Román, 2011) a los publicados originalmente. La diferencia en los datos probablemente radica, no tanto en una falta de consenso en la conceptualización teórica sobre qué es el efecto bruto del centro sino en el modo en que cómo se mide dicho efecto, es decir, en el hecho de que los estudios difieren en aspectos básicos, como la materia evaluada, la etapa educativa y la edad del alumnado, las características de los centros (titularidad y zona geográfica), los diseños muestrales empleados para seleccionar al alumnado participante y el número de niveles de agregación empleados en el análisis multinivel.

Existen bastantes datos que indican que el efecto bruto del centro es mayor en las materias científico-matemáticas que en las sociolingüísticas (Blanco-Bosco, 2011; Casas, Gamboa, y Piñeros, 2002; Cervini, 2002a, 2002b, 2012; Scheerens, 2016; UNESCOOREALC y LLECE, 2001, 2010; Zorrilla, 2009). Sin embargo, Carvallo (2006) y Cervini, Dari y Quiroz (2016) señalan lo contrario, y otros autores informan de diferencias muy pequeñas (Murillo y Román, 201 1), cuando no efectos similares en ambas materias (Cervini, 2006). Incluso dentro de una misma materia se estiman efectos diferentes en función de sus ejes de especificación. Peña-Suárez, Campillo-Álvarez, Santarén-Rosell y Muñiz (2012), con datos de PISA 2006 y empleando como variable dependiente los resultados en las competencias científicas, encuentran que el efecto bruto del centro es mayor cuando la variable a predecir son las capacidades 
científicas (identificar cuestiones científicas; explicar fenómenos científicamente; y usar pruebas científicas) que cuando son las actitudes hacia la ciencia (interés por la ciencia; y apoyo a la investigación científica.

lgualmente, los datos disponibles no presentan una pauta clara que relacione el tamaño del efecto bruto del centro y la edad o etapa educativa del estudiante. Una proporción de la investigación señala que a medida que aumenta la edad disminuye ligeramente el tamaño del efecto (Scheerens et al., 2013). Sin embargo, Cervini (2010) encuentra mayor efecto en educación secundaria que en primaria, y no faltan estudios que informan de que el efecto es similar en ambas etapas (Cervini, 2009) y que, por tanto, no encuentran relación entre la edad del alumnado y el tamaño del efecto bruto del centro (Murillo y Román, 2011 1). Las variaciones a la hora de estimar el efecto bruto del centro también se observan en función de algunas características de la escuela como la titularidad o la zona geográfica. Piñeros y Rodríguez Pinzón (1998) encuentran que el coeficiente de correlación intraclase es mayor en los centros privados que en los públicos, aunque la revisión de Casas et al. (2002) no permite confirmar la afirmación anterior. Por su parte, Reimers (2002) señala que el efecto bruto en las escuelas rurales e indígenas es entre dos y tres veces mayor que en las escuelas urbanas.

Por último, el tamaño del efecto bruto del centro también parece depender de características metodológicas del estudio, tales como el diseño muestral empleado, el modo en que se define o instrumentaliza la variable dependiente y el número de niveles incluidos en el análisis jerárquico-lineal. Carvallo (2006), trabajando con edades similares, señala un efecto menor en estudios censales que en estudios muestrales, y Cervini (2004a, 2004b), después de excluir los centros más pequeños de su estudio, encuentra una disminución del efecto bruto, probablemente por eliminar parte de las variaciones vinculadas a las escuelas rurales y remotas. Por su parte, Casas et al. (2002), en un estudio longitudinal con datos colombianos, encuentran una disminución del tamaño del efecto asociada a un cambio en el tipo de examen, lo que indica que una modificación en la especificación de la variable dependiente puede afectar al poder discriminatorio de las pruebas a nivel de centro. Finalmente, la evidencia sobre las variaciones del efecto bruto del centro en función del número de niveles de los modelos jerárquico-lineales parece bien documentada. Cervini (2006, 2010, 2012), comparando el tamaño del efecto en modelos de entre dos y cuatro niveles, encuentra que a medida que aumenta el número de niveles disminuye el tamaño del efecto del centro como consecuencia lógica de la distribución de la varianza entre-centros en niveles de agregación superiores (país, provincia) o inferiores (aula), y Scheerens (2016), en una revisión que compara estudios que incluyen datos del nivel estudiante, profesor/aula y escuela, señala que la omisión de niveles intermedios (profesor/aula) conduce a una sobreestimación del efecto del siguiente nivel superior, como podría ser el caso de las escuelas.

En general se ha asumido que los sistemas educativos más equitativos tienden a mostrar efectos brutos entre centros más pequeños (OCDE, 2002). Sin embargo, esto no es del todo cierto, un sistema educativo podría presentar diferencias absolutas pequeñas entre centros, pero si todas las variaciones estuvieran determinadas por el contexto sociológico y demográfico la capacidad de mejora de los centros sería nula o muy limitada. El caso opuesto sería aquel sistema educativo que mostrara grandes diferencias entre sus centros, pero donde la influencia de los antecedentes sociológicos sobre los resultados fuera muy débil, ya que en este caso las prácticas educativas de los centros tendrían mayor capacidad compensatoria. Por ello, otra forma tradicional de estimar la inequidad de los sistemas educativos es calcular el grado de asociación entre los desempeños escolares y las características del contexto educativo, entendiendo que la relación entre los antecedentes socioeconómicos y culturales del alumnado y su rendimiento académico es una medida de la equidad con la que un 
sistema educativo distribuye las oportunidades de aprendizaje y del potencial compensatorio de cada escuela (OECD, 2010). Para su estimación se especifica un modelo, generalmente jerárquico-lineal, donde las variables de contexto se incluyen en diferentes niveles, y que permite estimar el efecto neto de las escuelas, que operativamente se define como la proporción de varianza entre centros que queda por explicar una vez controlados los factores antecedentes (Rodríguez-Jiménez y Murillo, 2011).

Existen abundantes datos que apoyan la relevancia de los factores sociodemográficos para explicar las diferencias en los resultados de los centros (OECD, 2002, 2004, 2007, 2010, 2013, 2016; Sirin, 2005). Sin embargo, al igual que ocurriera al hablar del efecto bruto, la estimación de la potencia explicativa de las variables de contexto varía de unos estudios a otros. Por ejemplo, SERCE, una vez detraídas las variables de ajuste, estimó los siguientes efectos netos de centro: $44 \%$ en Ciencias Naturales; $37 \%$ en Matemáticas; y $31 \%$ en Lectura (UNESCO-OREALC y LLECE, 2010). Sin embargo, Murillo y Román (2011) consideran muy elevados estos valores y reanalizan los datos encontrando que el efecto neto del centro oscila entre el 17\% (Lectura) y el $22 \%$ (Matemática). Por su parte, Cervini (2016), con la base de datos TERCE y un modelo jerárquico-lineal de tres niveles, aún rebaja más este efecto, situándolo entre el $11 \%$ en Matemáticas y el $7 \%$ en Lectura. Las razones de esta disparidad en las estimaciones parecen ser tres: la variable dependiente empleada, el número y la naturaleza de las variables independientes y el nivel o niveles de análisis en que son consideradas estas últimas variables.

En general los datos disponibles indican que los factores demográficos, económicos y culturales tienen mayor impacto cuando la variable criterio es la lengua vernácula que cuando se trata de una materia científicomatemática (Cervini, 2002b, 2009, 2012; Cervini et al., 2016). Sin embargo, este resultado no siempre se replica en estudios latinoamericanos ya que Cervini (2006, 2010) y Murillo y Román (2011) encuentran efectos similares en Lenguaje y Matemáticas, y Zorrilla (2009) encuentra que el efecto es mayor en Matemáticas. En todo caso, parece que cuando la variable dependiente es una lengua extranjera (por ejemplo, inglés en los países hispanohablantes) el efecto del contexto social y demográfico sobre los resultados es mayor que en cualquier otra materia (Consejería de Educación y Ciencia del Principado de Asturias, 2007).

Una segunda fuente variación en la proporción de varianza explicada por los modelos de eficacia relativa es el número y la naturaleza de las variables antecedentes. En general, la variable con mayor fuerza suele ser un índice construido, generalmente tipificado, que resume el nivel socioeconómico y cultural de la familia del alumnado o, en su defecto, variables que describen el nivel de estudios y profesiones de los progenitores (Peña Suárez, Fernández-Alonso, y Muñiz, 2009; Sirin, 2005). Doneschi (2017) en un estudio de dos niveles que compara la inclusión progresiva de variables de contexto en cada nivel, señala que el índice socioeconómico es la variable con mayor capacidad para dar cuenta de las diferencias en los resultados, mientras que otros estudios informan que el nivel socioeconómico explica aproximadamente la mitad de la varianza entre centros (Casas et al., 2002; Rodríguez-Jiménez y Murillo, 2011 ). También han mostrado su relevancia algunas variables dicotómicas, como el género, la escolarización temprana, la lengua materna o la condición de emigrante, y otras medidas en escala ordinal o continua, como el número de libros en el hogar, las posesiones materiales y las características de la vivienda (Murillo y Román, 2011 ; UNESCO-OREALC y LLECE, 2000). Igualmente, en los estudios Latinoamericanos parecen importantes dos variables más, que generalmente no se consideran en la investigación con países desarrollados: ser indígena y compatibilizar trabajo y estudios (UNESCO-OREALC y LLECE, 2016a). Mención especial merece el tratamiento de la repetición escolar, ya que es una variable con importantes efectos sobre el rendimiento académico incluso una vez descontadas el resto de variables de contexto 
ya mencionadas (Gobierno del Principado de Asturias, 2016). Evidentemente en los diseños de investigación descriptivos expost facto la repetición es una variable antecedente, sin embargo, también es una variable modificable por los centros, ya que es una decisión que mayoritariamente se toma en los claustros escolares. Por tanto, considerarla o no como variable de contexto afectará a la proporción de varianza explicada por los modelos de ajuste.

Finalmente, el potencial de los factores de contexto para aquilatar las diferencias entre centros depende del nivel de análisis en el que se incluyen dichos factores. Cuando las variables se introducen únicamente a nivel de alumnado tienen menos incidencia, que cuando se manejan como promedios de aula o centro (Cervini, 2002a, 2002b, 2004b, 2006, 2012; Cervini et al., 2016). Esta limitación de los antecedentes sociológicos para explicar la varianza intra-centros está relacionada con el hecho de que la historia escolar, el rendimiento previo y algunas variables psicológicas, como la capacidad, la motivación, las expectativas o los estilos de aprendizaje, tienen más efecto sobre los resultados individuales que las variables de contexto económico y cultural (Fernández Alonso, Suárez-Álvarez, y Muñiz, 2015, 2016 ; Rodríguez, Piñeiro, Regueiro, Estevez, y Val, 2017; Suárez-Álvarez, Fernández-Alonso, y Muñiz, 2014). No obstante, los modelos que dan cuenta de un mayor porcentaje de varianza son aquellos que consideran conjuntamente las variables de ajuste en dos o más niveles de análisis, lo que muestra la superioridad de los modelos jerárquico-lineales para explicar los resultados educativos (Fernández-Alonso, Álvarez-Díaz, Suarez-Alvarez, y Muñiz, 2017). En general la reducción de la varianza es mayor en el nivel de centro (o aula) que en el individual (Ferrão y Fernandes, 2001 ; PeñaSuárez et al., 2012), si bien aquí también se advierten notables diferencias en la potencia de los modelos, en algunos casos la varianza explicada está por debajo del 50\% (Cervini, 2002a, 2002b, 2012; Murillo y Román, $2011)$, en otros se sitúa en torno al $60 \%$ (Cervini, 2010; Rodríguez-Jiménez y Murillo,
2011 ), y en las soluciones más potentes los factores antecedentes dan cuenta del 80\% (Cervini et al., 2016) y hasta del $90 \%$ de las diferencias entre los centros (Doneschi, 2017).

En este contexto el objetivo general del presente estudio es analizar los efectos de los centros educativos sobre el rendimiento académico en Latinoamérica, empleando para ello los datos obtenidos en la evaluación TERCE. Este objetivo general se concreta en cuatro objetivos específicos: a) ¿Cuál es el efecto global de los centros escolares en el rendimiento académico de los estudiantes de Latinoamérica?, b) ¿QQué porcentaje de la varianza total y de la varianza entre los centros está asociada al nivel socioeconómico y cultural de los estudiantes? c) ¿¿Qué incremento experimenta la proporción de la varianza explicada cuando se incluyen otras variables de ajuste?, y d) ¿Cuál es el efecto neto de los centros escolares latinoamericanos sobre el rendimiento académico cuando se controlan las variables de ajuste?

\section{MÉTODO}

\section{PARTICIPANTES}

La población objetivo se definió como el alumnado matriculado en $6^{\circ}$ de educación primaria en el curso 2013 en los 15 países participantes y el estado de Nuevo León (México). Dentro de cada país la muestra fue seleccionada siguiendo un diseño bietápico y estratificado propio de este tipo de comparaciones internacionales (Joncas y Foy, 2012). En la primera etapa los centros participantes se seleccionaron con una probabilidad proporcional a su tamaño, y en la segunda etapa se eligió un grupo aula del centro mediante un muestreo aleatorio simple. En el momento de liberar los datos, LLECE construyó tres bases (una por materia evaluada: Ciencias Naturales, Matemáticas y Lectura) y excluyó de cada base de datos al alumnado que, por diversas razones, no hubiese respondido a la materia correspondiente, lo que hizo que en la práctica cada matriz de datos presentara tamaños muestras distintos, aunque muy similares. La tabla 1 recoge el 
número de estudiantes y de centros finalmente incluidos por materia, los cuales representan a una población de prácticamente 9 millones de estudiantes. La media de edad del alumnado es de 12,4 años (DT = ,94). El 69,4\% asiste a un centro público; el $50,3 \%$ son mujeres; y el $20,4 \%$ ha repetido al menos un curso en el momento de la aplicación de la prueba.

Tabla 7 .

Distribución de la muestra por materia evaluada

\begin{tabular}{|l|c|c|c|}
\hline & $\begin{array}{c}\text { Ciencias } \\
\text { Naturales }\end{array}$ & Matemática & Lectura \\
\hline Estudiantes & 61938 & 63750 & 60949 \\
Escuelas & 2955 & 2934 & 2954 \\
\hline
\end{tabular}

\section{PROCEDIMIENTO}

El estudio es implementado por el LLECE en cooperación con las coordinaciones de los sistemas educativos de los países participantes, quienes otorgan los permisos de acceso al personal experto y externo al centro encargado de la aplicación de las pruebas, conservando la confidencialidad de los participantes (UNESCO-OREALC, 2016 b). La aplicación se desarrolló en dos jornadas, la primera dedicada a Lectura y Escritura y la segunda a Matemáticas y Ciencias. La evaluación de cada materia ocupó entre 45 y 60 minutos, con un descanso de 30 minutos. El cuestionario de contexto del alumnado, de 45 minutos de duración, se aplicó al final de la segunda jornada, tras un receso de 15 minutos. El primer día se entregaron los cuestionarios para el centro, profesorado y familias, y se recogieron al final de la segunda jornada. La evaluación se desarrolló de acuerdo con los estándares éticos de la UNESCO. Las familias de los estudiantes seleccionados para participar en la evaluación fueron informadas por las direcciones escolares, y pudieron decidir o no sobre la participación de sus hijos en el estudio.

\section{INSTRUMENTOS}

Se emplearon pruebas cognitivas de conocimientos en las tres materias mencionadas, y las puntuaciones en dichas pruebas se emplean como variables criterio en este estudio. Por su parte, las variables de ajuste se extraen de las respuestas a los cuestionarios de contexto ya reseñados. Lo instrumentos, creados por expertos en varios campos de estudio contratados LLECE y validados en un estudio piloto, cumplen con los criterios psicométricos comunes a todo procedimiento de evaluación (Martínez-Arias, 2010).

\section{Test de rendimiento académico}

La prueba de Ciencias constaba de 92 ítems de elección múltiple y abiertos de respuesta corta agrupados en seis bloques diseñados para cubrir una tabla de especificaciones bivariada que evaluaba tres procesos cognitivos (Reconocimiento de información y conceptos; Comprensión y aplicación de conceptos; y Pensamiento científico y resolución de problemas), y cinco dominios de conocimientos (Salud; Seres vivos; Ambiente; La tierra y el sistema solar; y Materia y energía). La prueba de Matemáticas contenía 98 ítems de los dos formatos mencionados y distribuidos en seis bloques de 16 ó 17 ítems cada uno. Las especificaciones de contenido se organizaron en una matriz de doble entrada: tres procesos cognitivos (Reconocimiento de objetos y elementos; Solución de problemas simples; y Solución de problemas complejos), y cinco dominios de conocimientos (Numérico; Geométrico; Medición; Estadístico; y Variación) (UNESCO-OREALC, 2016b). Finalmente, la prueba de Lectura constaba de 96 ítems de elección múltiple y respuesta construida asignados a seis clústers de 16 ítems cada uno, y distribuidos sobre una matriz de especificaciones que evaluaba tres procesos cognitivos (Recuperación literal de información; Realización de inferencias; y Lectura crítica) y dos dominios de conocimientos (Comprensión de textos; y Dominio metalingüístico y teórico) (UNESCO-OREALC, $2016 \mathrm{~b}$ ). 
Dado que los estudiantes no pueden responder al conjunto de ítems en el tiempo de evaluación disponible, los bloques de ítems se distribuyeron en seis modelos de cuadernillos por cada materia siguiendo un diseño matricial de bloques incompletos parcialmente balanceado (Fernández-Alonso y Muñiz, 201 1) y cada estudiante respondió a un cuadernillo que contenía entre 31 y 33 ítems. El promedio del coeficiente alfa de Cronbach de los cuadernillos fue de ,72 en Ciencias Naturales; ,80 en Matemáticas; y ,85 en Lectura. La puntuación fue calculada mediante la metodología de valores plausibles, por ser la más eficiente para recuperar los parámetros poblaciones en las evaluaciones de sistemas educativos (Mislevy, Beaton, Kaplan, y Sheehan, 1992; OECD, 2009). Las puntuaciones individuales fueron expresadas en una escala con media 700 puntos y desviación típica 100 (UNESCO-OREALC, 2016b).

\section{Variables de control o ajuste}

Para describir las características sociológicas del alumnado se han considerado seis variables, cinco de ellas dicotómicas: Género $(1=$ ser mujer); Indígena ( 1 = pertenecer a una etnia indígena); Repetidor ( 1 = haber repetido algún curso durante la escolaridad); Trabajar (1= el estudiante trabaja y recibe una remuneración por esa actividad); y Cuaderno $(1=$ el estudiante posee cuaderno para anotaciones). La sexta variable es una estimación del Nivel socioeconómico y cultural del alumnado (ISEC), un índice estandarizado por TERCE y compuesto por 17 ítems que recababan información sobre el nivel educativo de los progenitores, tipo de trabajo que realizan, ingresos familiares, disponibilidad de material de lectura del hogar y bienes y servicios del barrio donde se ubica la vivienda. Los valores del alfa de Cronbach del índice oscilan entre ,8 y ,9 según el país (UNESCO-OREALC, $2016 \mathrm{~b}$ ).

Dentro de las características del contexto social y demográfico de la escuela se consideraron cinco variables, dos de ellas dicotómicas: Titularidad $(0=$ centro público; 1 = centro privado) y Ruralidad ( $1=$ centro rural). Los recursos de la escuela se estimaron mediante el Nivel de Infraestructura del centro, un índice estandarizado elaborado con 19 ítems del cuestionario para las direcciones que evaluaba las instalaciones, equipamientos y servicios de escuela. Los valores del alfa de Cronbach del índice oscilan entre ,7 y ,9 según el país (UNESCO-OREALC, 2016b). Las dos últimas variables fueron el Nivel socioeconómico y cultural del centro y del país, calculados como el promedio del ISEC por centro y por país.

\section{ANÁLISIS DE DATOS}

Se emplearon modelos jerárquico-lineales, que previenen contra errores de inferencia estadística como el sesgo de segregación o la heterogeneidad de la regresión, y permiten separar los efectos de la escuela de los factores propios de los estudiantes (MartínezArias, Gaviria-Soto, y Castro-Morera, 2009). Inicialmente, para cada competencia y país (incluida la muestra completa) se ajustó una secuencia de tres modelos de 2 niveles (alumnado y escuela): Modelo I, sin variables predictoras; Modelo II, que incluye el ISEC en ambos niveles (estudiante y centro); y Modelo III, que añade al modelo anterior el resto de variables de contexto (Nivel estudiante: género, indígena, repetidor, trabajar, cuaderno; Nivel centro: titularidad, ruralidad, nivel de infraestructura). En total se especificaron 153 ajustes (3 modelos $\times 3$ competencias $x$ 17 muestras). Con el Modelo I se estimó el coeficiente de correlación intraclase (ICC) para la determinación del efecto bruto de los centros, y con los Modelos II y III se calculó la proporción de varianza explicada, entendida como la reducción de varianza (total y por nivel) que experimenta el Modelo I al incluir las variables de control (Efecto ISEC) y el efecto neto del centro, entendido como el porcentaje de varianza contenida en el nivel de centros de los Modelos II y III.

Adicionalmente, empleando la muestra completa, los modelos I, II y III se especificaron para un análisis jerárquico-lineal de 3 niveles (alumnado, escuela y país) lo que dio lugar a 9 ajustes más (3 modelos $\times 3$ competencias). En las tablas de resultados estos modelos se identificarán como TERCE 3-N, y permiten 
estimar los efectos ya mencionados, pero segregando la varianza al nivel de país. El análisis se realizó con HLM 7.01 (Raudenbush, Bryk, Cheong, Congdon, y du Toit, 2011 1), empleando el método de estimación de Máxima Verosimilitud y tomando como variable dependiente los cinco valores plausibles de cada competencia. En los modelos ajustados con la base completa los casos fueron ponderados empleando los pesos senatoriales ofrecidos por TERCE, de modo que, en cada país, la suma de los pesos equivalía a 5000 estudiantes escolarizados en 200 escuelas. En los modelos ajustados para cada país individualmente considerado, se emplearon los pesos muestrales de modo que la suma de estos pesos equivalía a la matrícula total de estudiantes de $6^{\circ}$ de educación primaria y al número total de centros de educación primaria de cada país (UNESCO-OREALC, 2016b).

El rango de casos perdidos en las variables osciló entre el $2 \%$ y el $12 \%$, y para su recuperación se empleó una estrategia en dos pasos. Inicialmente los casos incompletos fueron imputados por la media del sujeto y, a continuación, los datos totalmente perdidos fueron recuperados mediante el método iterativo (método EM con variables auxiliares) que implementa el módulo Missing Value Análisis del programa SPSS 15.
Fernández-Alonso,Suárez-ÁlvarezyMuñiz(2012) encontraron que esta estrategia de dos pasos es la que mejor recupera los datos poblacionales cuando el mecanismo de la pérdida no es aleatorio y el porcentaje de datos perdidos es similar al registrado en TERCE.

\section{RESULTADOS}

La Tabla 2 muestra la distribución de la varianza en los niveles alumnado y centro para cada país y materia evaluada, así como el porcentaje de la varianza total contenida en el nivel de centro, expresada como el coeficiente de correlación intraclase (ICC). El porcentaje de varianza total atribuido al nivel de país, que no está recogida en el modelo TERCE 3-N, fue del 13\% Ciencias Naturales, 26\% en Matemáticas y $17 \%$ en Lectura. En todo caso el modelo TERCE 3-N muestra una importante reducción del efecto bruto del centro cuando se compara con el modelo de dos niveles (TERCE 2-N), si el análisis no hubiese considerado el nivel de país el ICC sería entre 10 y 23 puntos porcentuales mayor. Para el conjunto de la muestra Ciencias es la materia con mayor ICC (33\%), mientras que Lectura y Matemáticas presentan 5 puntos porcentuales menos.

Tabla 2 .

Modelo I: Efecto bruto sin variables predictoras. Distribución de la varianza por nivel de análisis y efecto bruto del centro expresado como porcentaje de varianza entre centros (varianza nivel 2)

\begin{tabular}{|c|c|c|c|c|c|c|c|c|c|}
\hline & \multicolumn{3}{|c|}{ Ciencias Naturales } & \multicolumn{3}{|c|}{ Matemática } & \multicolumn{3}{|c|}{ Lectura } \\
\hline & $\begin{array}{c}\text { Varianza } \\
\text { Nivel } 1\end{array}$ & $\begin{array}{c}\text { Varianza } \\
\text { Nivel } 2\end{array}$ & $\begin{array}{l}\text { Efecto } \\
\text { bruto }\end{array}$ & $\begin{array}{c}\text { Varianza } \\
\text { Nivel } 1\end{array}$ & $\begin{array}{c}\text { Varianza } \\
\text { Nivel } 2\end{array}$ & $\begin{array}{l}\text { Efecto } \\
\text { bruto }\end{array}$ & $\begin{array}{c}\text { Varianza } \\
\text { Nivel } 1\end{array}$ & $\begin{array}{c}\text { Varianza } \\
\text { Nivel } 2\end{array}$ & $\begin{array}{l}\text { Efecto } \\
\text { bruto }\end{array}$ \\
\hline TERCE (2-N) & 5943 & 4443 & $43 \%$ & 5119 & 5428 & $51 \%$ & 5787 & 4782 & $45 \%$ \\
\hline TERCE (3-N) & 5903 & 3560 & $33 \%$ & 4926 & 3005 & $28 \%$ & 5822 & 3019 & $28 \%$ \\
\hline Argentina & 6256 & 3421 & $35 \%$ & 4847 & 2793 & $37 \%$ & 6541 & 3931 & $38 \%$ \\
\hline Brasil & 5991 & 3323 & $36 \%$ & 4062 & 3480 & $46 \%$ & 6409 & 2980 & $32 \%$ \\
\hline Chile & 9569 & 3412 & $26 \%$ & 8226 & 2399 & $23 \%$ & 8087 & 2060 & $20 \%$ \\
\hline Colombia & 6149 & 2767 & $31 \%$ & 4201 & 2744 & $40 \%$ & 5401 & 3807 & $41 \%$ \\
\hline
\end{tabular}


Tabla 2. (Continuación)

Modelo I: Efecto bruto sin variables predictoras. Distribución de la varianza por nivel de análisis y efecto bruto del centro expresado como porcentaje de varianza entre centros (varianza nivel 2)

\begin{tabular}{|c|c|c|c|c|c|c|c|c|c|}
\hline & \multicolumn{3}{|c|}{ Ciencias Naturales } & \multicolumn{3}{|c|}{ Matemática } & \multicolumn{3}{|c|}{ Lectura } \\
\hline & $\begin{array}{c}\text { Varianza } \\
\text { Nivel } 1\end{array}$ & $\begin{array}{c}\text { Varianza } \\
\text { Nivel } 2\end{array}$ & $\begin{array}{l}\text { Efecto } \\
\text { bruto }\end{array}$ & $\begin{array}{c}\text { Varianza } \\
\text { Nivel } 1\end{array}$ & $\begin{array}{c}\text { Varianza } \\
\text { Nivel } 2\end{array}$ & $\begin{array}{l}\text { Efecto } \\
\text { bruto }\end{array}$ & $\begin{array}{c}\text { Varianza } \\
\text { Nivel } 1\end{array}$ & $\begin{array}{c}\text { Varianza } \\
\text { Nivel } 2\end{array}$ & $\begin{array}{l}\text { Efecto } \\
\text { bruto }\end{array}$ \\
\hline Costa Rica & 6102 & 1852 & $23 \%$ & 4561 & 1456 & $24 \%$ & 5255 & 1586 & $23 \%$ \\
\hline Ecuador & 5878 & 3778 & $39 \%$ & 4164 & 3140 & $43 \%$ & 4954 & 3268 & $40 \%$ \\
\hline Guatemala & 4270 & 2909 & $41 \%$ & 3553 & 2764 & $44 \%$ & 4279 & 2643 & $38 \%$ \\
\hline Honduras & 4589 & 3131 & $41 \%$ & 3326 & 2815 & $46 \%$ & 4018 & 2343 & $37 \%$ \\
\hline México & 5638 & 2445 & $30 \%$ & 7715 & 2810 & $27 \%$ & 6365 & 3358 & $35 \%$ \\
\hline Nicaragua & 3674 & 2546 & $41 \%$ & 2361 & 1630 & $41 \%$ & 4315 & 2350 & $35 \%$ \\
\hline Nuevo León & 6065 & 2227 & $27 \%$ & 8979 & 2109 & $19 \%$ & 7031 & 2227 & $24 \%$ \\
\hline Panamá & 5473 & 3558 & $39 \%$ & 3166 & 2220 & $41 \%$ & 5481 & 3340 & $38 \%$ \\
\hline Paraguay & 4927 & 4143 & $46 \%$ & 3601 & 4055 & $53 \%$ & 5116 & 3420 & $40 \%$ \\
\hline Perú & 4696 & 4195 & $47 \%$ & 5302 & 6943 & $57 \%$ & 4678 & 6601 & $59 \%$ \\
\hline $\begin{array}{l}\text { Rep. } \\
\text { Dominicana }\end{array}$ & 4368 & 1171 & $21 \%$ & 2670 & 612 & $19 \%$ & 4357 & 1439 & $25 \%$ \\
\hline Uruguay & 8827 & 2927 & $25 \%$ & 8301 & 2074 & $20 \%$ & 8213 & 2811 & $25 \%$ \\
\hline
\end{tabular}

Los datos del Modelo I señalan que en todos los países el efecto bruto de la escuela es significativo, aunque no es menos cierto que se advierten importantes variaciones en la magnitud del ICC de cada país. Perú presenta el mayor porcentaje de variación entre centros, llegando a ser incluso superior a la varianza dentro de los centros en Matemáticas (57\%) y Lectura (59\%), seguido de Paraguay donde el coeficiente de correlación intraclase supera el $45 \%$ en dos de las tres áreas. En Argentina, Ecuador, Guatemala, Honduras, Nicaragua y Panamá los ICC de todas las materias están en el rango ,35 -, 45. En el extremo contrario, es decir, los países con un tamaño del efecto del centro menor son Chile, Costa Rica, República Dominicana, Uruguay y el estado de Nuevo León, donde el ICC no alcanza el 30\% en ninguna materia.

Por otro lado, las estimaciones del efecto bruto de los centros son bastante estables.
La correlación de los ICC de los países es de ,85 entre Matemáticas y Lectura; ,82 entre Ciencias y Lectura; y ,95 entre Ciencias y Matemáticas, lo que indica que, dentro de cada país, las diferencias entre sus centros tienden a ser similares independientemente de la materia considerada.

La tabla 3 muestra el porcentaje de varianza total y por nivel explicada por el Modelo II (ISEC del alumnado y del centro) y el Modelo III (con todas las variables de control) para el conjunto de países. Lectura y Matemáticas presentan una asociación entre resultados y variables de ajuste similar, explicando en torno al $30 \%$ de la varianza total, mientras que en el caso de Ciencias este porcentaje se ubica sobre el $20 \%$. La fuerza explicativa del ISEC es mucho mayor en el nivel 2, ya que en el conjunto del estudio (Modelo II, TERCE 3-N) el ISEC da cuenta del $65 \%$ de las diferencias inter-centros en Lectura y en torno al $35 \%$ en 
Ciencias Naturales y Matemáticas, mientras que los porcentajes de varianza explicada en el nivel individual no superan el $2 \%$. Cuando se incluyen el resto de las variables de contexto (Modelo III, TERCE 3-N) la ganancia explicativa es más discreta en Ciencias, donde la varianza total explicada se incrementa un $2 \%$, que en Lectura y Matemáticas ( $4 \%$ y $7 \%$ de incremento respectivamente). Por su parte, los incrementos de la varianza explicada en el nivel 2 están entre el $3 \%$ y el $6 \%$, y entre el $1 \%$ y el $3 \%$ en el nivel 1 .

Porcentaje de varianza total y por niveles explicada por el Modelo II (ISEC en ambos niveles) y el Modelo III (todas las variables de ajuste)

\begin{tabular}{|c|c|c|c|c|c|c|c|c|c|}
\hline & \multicolumn{3}{|c|}{ Ciencias Naturales } & \multicolumn{3}{|c|}{ Matemática } & \multicolumn{3}{|c|}{ Lectura } \\
\hline & Nivel 1 & Nivel 2 & Total & Nivel 1 & Nivel 2 & Total & Nivel 1 & Nivel 2 & Total \\
\hline \multicolumn{10}{|c|}{$\begin{array}{l}\text { Dos Niveles } \\
\text { (TERCE 2-N) }\end{array}$} \\
\hline Modelo II & $1 \%$ & $50 \%$ & $22 \%$ & $1 \%$ & $48 \%$ & $25 \%$ & $2 \%$ & $69 \%$ & $32 \%$ \\
\hline $\begin{array}{l}\text { Modelo III } \\
\text { Tres Nivel } \\
\text { (TERCE 3- }\end{array}$ & $3 \%$ & $52 \%$ & $24 \%$ & $2 \%$ & $52 \%$ & $28 \%$ & $4 \%$ & $72 \%$ & $35 \%$ \\
\hline Modelo II & $2 \%$ & $36 \%$ & $20 \%$ & $1 \%$ & $35 \%$ & $22 \%$ & $1 \%$ & $65 \%$ & $29 \%$ \\
\hline Modelo III & $4 \%$ & $39 \%$ & $22 \%$ & $2 \%$ & $41 \%$ & $29 \%$ & $4 \%$ & $68 \%$ & $33 \%$ \\
\hline
\end{tabular}

Tabla 4

Porcentaje de varianza total explicada por los Modelos II y III en cada país

\begin{tabular}{|c|c|c|c|c|c|c|}
\hline & \multicolumn{3}{|c|}{ Modelo II } & \multicolumn{3}{|c|}{ Modelo III } \\
\hline & $\begin{array}{l}\text { Ciencias } \\
\text { Naturales }\end{array}$ & Matemática & Lectura & $\begin{array}{l}\text { Ciencias } \\
\text { Naturales }\end{array}$ & Matemática & Lectura \\
\hline Argentina & $20 \%$ & $16 \%$ & $25 \%$ & $27 \%$ & $20 \%$ & $32 \%$ \\
\hline Brasil & $26 \%$ & $30 \%$ & $26 \%$ & $30 \%$ & $31 \%$ & $28 \%$ \\
\hline Chile & $14 \%$ & $12 \%$ & $13 \%$ & $18 \%$ & $14 \%$ & $16 \%$ \\
\hline Colombia & $14 \%$ & $24 \%$ & $30 \%$ & $18 \%$ & $27 \%$ & $34 \%$ \\
\hline Costa Rica & $16 \%$ & $15 \%$ & $21 \%$ & $20 \%$ & $20 \%$ & $26 \%$ \\
\hline Ecuador & $13 \%$ & $11 \%$ & $32 \%$ & $16 \%$ & $15 \%$ & $36 \%$ \\
\hline Guatemala & $32 \%$ & $28 \%$ & $32 \%$ & $34 \%$ & $35 \%$ & $35 \%$ \\
\hline Honduras & $6 \%$ & $2 \%$ & $17 \%$ & $14 \%$ & $12 \%$ & $22 \%$ \\
\hline México & $18 \%$ & $17 \%$ & $28 \%$ & $22 \%$ & $22 \%$ & $32 \%$ \\
\hline Nicaragua & $8 \%$ & $6 \%$ & $20 \%$ & $10 \%$ & $12 \%$ & $22 \%$ \\
\hline Nuevo León & $19 \%$ & $13 \%$ & $21 \%$ & $20 \%$ & $17 \%$ & $25 \%$ \\
\hline Panamá & $26 \%$ & $23 \%$ & $31 \%$ & $29 \%$ & $27 \%$ & $33 \%$ \\
\hline Paraguay & $11 \%$ & $6 \%$ & $31 \%$ & $13 \%$ & $11 \%$ & $35 \%$ \\
\hline Perú & $32 \%$ & $33 \%$ & $47 \%$ & $34 \%$ & $38 \%$ & $51 \%$ \\
\hline Rep. Dominicana & $19 \%$ & $16 \%$ & $19 \%$ & $21 \%$ & $19 \%$ & $22 \%$ \\
\hline Uruguay & $19 \%$ & $17 \%$ & $27 \%$ & $27 \%$ & $21 \%$ & $32 \%$ \\
\hline
\end{tabular}


La tabla 4 muestra el porcentaje de varianza total explicada por los Modelos II y III en cada país. Perú es el país donde las variables antecedentes tienen mayor efecto sobre los resultados, estimándose que entre el $35 \%$ y el $50 \%$ de la varianza total se explica por factores de contexto. Guatemala presenta valores estables en torno al 35\% y en Brasil y Panamá los factores de contexto explican en torno a un tercio de las diferencias totales en todas las materias. En el extremo contrario, Chile, Costa Rica, Nicaragua, Honduras, República Dominicana y Nuevo León son los países donde el efecto de los antecedentes no supera el promedio del modelo TERCE 3-N en las tres competencias.
Mención especial merecen Ecuador y Paraguay, donde los factores de contextos explican el $35 \%$ de la varianza en Lectura y un porcentaje mucho más discreto en las competencias científico-matemáticas. En todo caso, la correlación entre el porcentaje de varianza total explicada por país en Ciencias y Matemáticas es más alta $(, 89)$, que la encontrada cuando se compara con la varianza en Lectura (la correlación LecturaMatemáticas es ,64 y Lectura-Ciencias ,54), lo que parece señalar que los países donde los factores de ajuste explican más diferencias en una materia tienden a presentar mayor capacidad explicativa en el resto.

Tabla 5.

Efecto Neto. Porcentaje de varianza entre centros sin explicar en los modelos II y III

\begin{tabular}{|c|c|c|c|c|c|c|}
\hline & \multicolumn{3}{|c|}{ Modelo II } & \multicolumn{3}{|c|}{ Modelo III } \\
\hline & $\begin{array}{l}\text { Ciencias } \\
\text { Naturales }\end{array}$ & Matemática & Lectura & $\begin{array}{l}\text { Ciencias } \\
\text { Naturales }\end{array}$ & Matemática & Lectura \\
\hline TERCE (2-N) & $28 \%$ & $36 \%$ & $21 \%$ & $27 \%$ & $34 \%$ & $20 \%$ \\
\hline TERCE (3-N) & $26 \%$ & $24 \%$ & $14 \%$ & $25 \%$ & $23 \%$ & $13 \%$ \\
\hline Argentina & $19 \%$ & $24 \%$ & $17 \%$ & $17 \%$ & $21 \%$ & $11 \%$ \\
\hline Brasil & $17 \%$ & $23 \%$ & $11 \%$ & $15 \%$ & $22 \%$ & $10 \%$ \\
\hline Chile & $15 \%$ & $12 \%$ & $10 \%$ & $12 \%$ & $11 \%$ & $8 \%$ \\
\hline Colombia & $21 \%$ & $21 \%$ & $18 \%$ & $19 \%$ & $19 \%$ & $15 \%$ \\
\hline Costa Rica & $10 \%$ & $13 \%$ & $7 \%$ & $10 \%$ & $12 \%$ & $5 \%$ \\
\hline Ecuador & $31 \%$ & $38 \%$ & $15 \%$ & $30 \%$ & $36 \%$ & $12 \%$ \\
\hline Guatemala & $13 \%$ & $22 \%$ & $11 \%$ & $12 \%$ & $17 \%$ & $9 \%$ \\
\hline Honduras & $37 \%$ & $45 \%$ & $24 \%$ & $34 \%$ & $40 \%$ & $22 \%$ \\
\hline México & $16 \%$ & $13 \%$ & $11 \%$ & $15 \%$ & $11 \%$ & $9 \%$ \\
\hline Nicaragua & $37 \%$ & $38 \%$ & $19 \%$ & $36 \%$ & $35 \%$ & $17 \%$ \\
\hline Nuevo León & $10 \%$ & $7 \%$ & $7 \%$ & $9 \%$ & $6 \%$ & $6 \%$ \\
\hline Panamá & $19 \%$ & $24 \%$ & $13 \%$ & $18 \%$ & $21 \%$ & $12 \%$ \\
\hline Paraguay & $39 \%$ & $50 \%$ & $15 \%$ & $40 \%$ & $49 \%$ & $12 \%$ \\
\hline Perú & $23 \%$ & $36 \%$ & $23 \%$ & $22 \%$ & $32 \%$ & $19 \%$ \\
\hline Rep. Dominicana & $8 \%$ & $5 \%$ & $10 \%$ & $7 \%$ & $5 \%$ & $9 \%$ \\
\hline Uruguay & $10 \%$ & $4 \%$ & $2 \%$ & $7 \%$ & $3 \%$ & $2 \%$ \\
\hline
\end{tabular}


La tabla 5 muestra el efecto del centro, esto es, el porcentaje de varianza entre centros sin explicar en los Modelos II y III después de controlar las variables antecedentes de cada modelo. El ajuste TERCE 3-N señala que, una vez detraído el influjo de todas las variables de contexto, quedaría por explicar el 25\% de las diferencias entre los centros en Ciencias Naturales; el 23\% en Matemáticas y el $13 \%$ en Lectura. Nótese que el modelo de dos niveles con datos completos (TERCE N-2) sobrevalora indebidamente el efecto neto, especialmente en Matemáticas y Lectura. La varianza sin explicar dentro de los países se ajusta al patrón general: en todos los casos (salvo República Dominicana) el efecto neto es mayor en Matemáticas que en Lectura. No obstante, se advierten importantes diferencias entre los países en el porcentaje de varianza por explicar: en República Dominicana, Uruguay y Nuevo León el margen de mejora de los centros una vez descontado las variables de control está por debajo del 10\% de la varianza total. En el extremo contrario Ecuador, Honduras, Nicaragua, Paraguay y Perú son los países que presentan mayor margen de mejora en el conjunto de las tres materias. En todo caso, los datos de los países de nuevo vuelven a estar altamente correlacionados, especialmente en Ciencias y Matemáticas $(r=96)$, lo que indica que los países que presentan efectos netos de centro más altos en una materia, tienden a presentar efectos más altos en las otras dos.

\section{DISCUSIÓN Y CONCLUSIONES}

Para responder a la primera pregunta del estudio se ha estimado el efecto bruto del centro en cada materia expresado como el ICC de un modelo jerárquico-lineal sin predictores, entendiéndose que un efecto bruto mayor se asocia a mayores diferencias entre los centros de un mismo país. Los datos señalan que no se han producido avances en la región en las dos últimas décadas. Los ICC ofrecido por PERCE en el año 1997 (UNESCOOREALC y LLECE, 2001) y el calculado para TERCE 2-N en el Modelo I (tabla 2) apenas han variado: en Lectura es similar en ambas ediciones y en Matemáticas se observa un descenso de 4 puntos porcentuales en el último estudio. Además, se observa que los ICC de los nueve países que participaron en los dos estudios han experimentado un crecimiento generalizado que se sitúa entre 2 y 22 puntos porcentuales según el país y la materia considerada. Al comparar los ICC de los países con datos en ambos estudios también se advierte que la correlación del efecto bruto del centro es de ,78 en Matemáticas y de ,81 en Lectura, es decir, que los países que presentaban mayores diferencias entre sus centros en la primera evaluación tienden a seguir mostrando más diferencias dos décadas después. Para interpretar coherentemente estas magnitudes debe tenerse en cuenta que el estudio PISA señala que los ICC de los países Latinoamericanos son superiores al promedio de los países desarrollados. Por ejemplo, son entre 2 y 3 mayores que el efecto bruto de España, y entre 4 y 5 veces más que los ICC de los sistemas educativos con menor efecto bruto (OECD, 2002, 2004, 2007, 2010, $2013,2016)$. Estos datos son coherentes con las estimaciones de Scheerens (2000), que concluye que en los países desarrollados el ICC está en torno al 10-15\%, mientras que en los países en vías de desarrollo el rango se eleva hasta el 30-40\%. Por tanto, la primera conclusión es que, desde que se dispone de datos comparables para la región, las diferencias brutas entre los centros en América Latina se mantienen estables, cuando no aumentan, y que aquellos países con mayores diferencias entre sus centros parece que no han logrado reverter la situación durante este tiempo.

Se ha apuntado que un efecto bruto alto entre centros no tiene porqué significar per se una mayor inequidad educativa, ya que el valor del ICC pudiera reflejar las decisiones políticas sobre la ordenación académica de los países (OECD, 2007). Por ello, el estudio se plantea dos preguntas referidas al efecto que las variables de contexto tienen sobre los resultados, ya que en un modelo sistémico estas variables no pueden ser manipuladas por los centros para mejorar sus rendimientos. Por tanto, en la medida en que las variables de ajuste expliquen más varianza el margen de 
mejora de los centros estará más limitado. Para el conjunto de los países, una vez se introduce el ISEC (Modelo II), la varianza entre centros del Modelo I se reduce en un 65\% en Lectura y un $35 \%$ en Matemáticas y Ciencias, estimación coherente con los datos reportados en otras investigaciones (Cervini, 2012; Cervini et al., 2016; Murillo y Román, 2011), que señalan que el efecto de ISEC es mayor en Lectura que en las materias científico-matemáticas. Según los datos de PISA la fuerza de la relación entre nivel socioeconómico y cultural y los resultados educativos de los países de la región es similar al promedio de la OCDE (OECD, 2002, 2004, 2007, 2010, 2013). En el Modelo III se introducen el resto de las variables de ajuste y, en términos generales, se aprecia una pequeña ganancia explicativa en las diferencias, lo que es coherente con los resultados de Doneschi (2017). En general, el ISEC ha mostrado buena capacidad para dar cuenta de la varianza de resultados lo que parece indicar que en TERCE se han superado las limitaciones que presentaba el ISEC construido en la segunda edición (SERCE), y que era la razón por la cual algunos autores señalaron que el índice socioeconómico del segundo estudio tenía menos impacto del esperado en los resultados de la prueba (Cervini, 2012; Murillo y Román, 2011). No obstante, se han observado tres casos (Nicaragua, Honduras y Paraguay) donde el ISEC presentaba un potencial débil o muy débil para explicar la varianza (en especial varianza entre los centros) en Matemáticas, si bien al introducir la colección completa de variables de ajuste todos ellos experimentan ganancias significativas, lo que demuestra la relevancia del uso de índices consistentes que resuman las características sociodemográficas de las familias para introducirlos en diferentes niveles de los modelos jerárquico-lineales (Peña Suárez et al., 2009).

La última pregunta del estudio pretendía estimar el efecto neto del centro, esto es el porcentaje de varianza entre centros que queda sin explicar en el Modelo III una vez se introducen todas las variables de ajuste. Este efecto neto se interpreta como el margen de mejora de los centros, es decir, un porcentaje más alto señala una mayor capacidad de la escuela para mejorar sus resultados más allá de los condicionantes sociológicos y compensar las desigualdades de acceso y permanencia mediante sus prácticas educativas. Los valores estimados del presente estudio (13\% en Lectura y $23 \%$ en Matemáticas) son coincidentes con los datos de Murillo y Román (2011), y algo más altos que los estimados por Cervini (2012, 2016), probablemente porque la eliminación de escuelas pequeñas y remotas operado por este último haya comprimido las diferencias entre el centros. Los efectos netos estimados también son consistentes con los ofrecidos por LLECE en el segundo estudio regional (UNESCO-OREALC y LLECE, 2010), ya que la correlación entre los efectos netos del centro por país de SERCE y del presente estudio son: ,81 en Lectura y ,82 en Matemáticas y Ciencias Naturales, lo que señala que en la última década el grado en que las variables antecedentes determinan los resultados de los centros se ha mantenido estable en la región.

Los resultados encontrados ayudan a comprender los fenómenos educativos en América Latina y tienen importantes implicaciones para la toma de decisiones políticas en la región más desigual del mundo en materia educativa (UNESCO-OREALC, $2016 a$, p. 89), en especial en lo relacionado con la ordenación académica de las enseñanzas; los procesos de escolarización del alumnado y criterios de asignación y admisión de estudiantes a los centros; y la distribución de recursos humanos y materiales (Duarte, Bos, Moreno, y Morduchowicz, 2013). Una lectura conjunta de los datos permite establecer al menos dos perfiles de países en función de las diferencias entre centros y del efecto de las variables de ajuste sobre sus resultados. El primer grupo estaría formado por Chile, Costa Rica, Nuevo León, República Dominicana y Uruguay, países que presentan un efecto bruto del centro relativamente pequeño, con ICC de hasta ,27 y un moderado impacto de las variables de ajuste sobre los resultados de entre ,14 a ,32. Es el grupo de países donde los resultados de los centros parecen ser más homogéneos y menos determinados por los antecedentes escolares, pero como contrapunto son los países donde el efecto 
neto del centro es menor (en general menos del 10\%). El segundo grupo está formado por Perú, Panamá, Brasil y Guatemala, que son países donde las diferencias brutas entre sus centros son mayores (el ICC se sitúa mayoritariamente en torno a 40) y donde el conjunto de variables de contexto explica mayor porcentaje de la varianza total inicial (en torno a un 30\%). En estos países los centros muestran mayor heterogeneidad en sus resultados y, según los datos aquí presentados parecen ser los sistemas educativos más desiguales.

El estudio tiene algunas limitaciones. En primer lugar, las medidas de desigualdad presentadas -efecto bruto, porcentaje de varianza explicada por factores de contexto y efecto neto del centro- son clásicas, pero no agotan las posibilidades de estudio de las desigualdades en educación. Sería posible, por ejemplo, construir indicadores de segregación que analicen cómo afecta al rendimiento el agrupamiento del alumnado en los centros en función de su origen social (OECD, 2016; Robert, 2007). La limitación más significativa en el diseño de la investigación es la falta de una medida del logro previo, que permita detraer varianza fundamentalmente del nivel intracentros. Intentando aliviar esto se ha utilizado la variable de repetición como un indicador de la historia escolar con dificultades de aprendizaje o de permanencia en el sistema educativo, sin embargo, no hay duda de que una medida de rendimiento previo es el mejor predictor del rendimiento del alumnado individualmente considerado (Fernández-Alonso et al., 2015, 2017). Las futuras líneas de investigación se orientan al estudio de los residuales de centros a la luz de los modelos ajustados y el estudio de los factores de proceso educativo que pudieran dar cuenta del porcentaje de varianza no explicado en los modelos aquí presentados, tales como los estilos parentales (Osorio y González-Cámara, 2016), o las actitudes de los profesores (Álvarez-Martino et al., 2016; Cunha et al., 2015), por citar sólo dos de las muchas posibles. Se ha advertido que, en el modelo que predice el resultado en Matemáticas, tres países presentaban alteraciones muy importantes en el valor del coeficiente de regresión del ISEC dependiendo del uso o no de los pesos muestrales, lo que parece demandar análisis adiccionales de corte metodológico para estudiar cómo los pesos muestrales influyen sobre la relación entre el ISEC y los resultados dentro de los países. Finalmente, existen evidencias que señalan que las diferencias entre los centros de un país pueden ser el resultado de las tradiciones y las circunstancias socio históricas asociadas a la propia construcción y estructura del sistema educativo (Foces Gil, 2015), por lo que cabría la posibilidad de analizar si la organización y ordenación de las enseñanzas de los países están vinculadas a las diferencias en el desempeño de los centros educativos.

\section{Conflicto de intereses}

Los autores declaran no tener ningún conflicto de intereses.

\section{REFERENCIAS}

Álvarez-Martino, E., Álvarez, M., Castro, P., Campo, M. A., y González-Mesa, C. (2016). Teacher's perception of disruptive behaviour in the classrooms. Psicothema, 28(2), 174-180. doi: $10.7334 /$ psicothema2015.215

Blanco-Bosco, E. (2009). La desigualdad de resultados educativos. Aportes a la teoría desde la investigación sobre eficacia escolar. Revista Mexicana de Investigación Educativa, 14(43), $1019-1049$.

Blanco-Bosco, E. (2011). Los límites de la escuela: educación, desigualdad y aprendizajes en México. México D.F.: El Colegio de México, Centro de Estudios Sociológicos.

Carvallo, M. (2006). Factores que afectan el desempeño de los alumnos mexicanos en edad de educación secundaria: un estudio dentro de la corriente de eficacia escolar. Revista Iberoamericana sobre Calidad, Eficacia y Cambio en Educación, 4(3), 30-53.

Casas, A., Gamboa, L. F., y Piñeros, L. J. (2002). El efecto escuela en Colombia, 1999-2000. Bogotá: Universidad del Rosario.

Castro-Aristizabal, G., Castillo-Caicedo, M., y Mendoza-Parra, J. (2016). Principales determinantes en la adquisición de competencias en América Latina: Un análisis multinivel a partir de los resultados en PISA 2012. Cali: Pontificia Universidad Javeriana. doi:10.2139/ ssrn.2744657

Cervini, R. (2002a). Desigualdades en el logro académico y reproducción cultural en Argentina. Revista Mexicana de Investigación Educativa, 7(16), 445-500. 
Cervini, R. (2002b). Desigualdades socioculturales en el aprendizaje de matemática y lengua de la educación secundaria en Argentina: Un modelo de tres niveles. Revista Electrónica de Investigación y Evaluación Educativa, 8(2), 135-158.

Cervini, R. (2004a). Influencia de los factores institucionales sobre el logro en matemática de los estudiantes en el último año de la educación media de Argentina- Un modelo de tres niveles. Revista Electrónica Iberoamericana sobre Calidad, Eficacia y Cambio en Educación, 2(1), $1-24$.

Cervini, R. (2004b). Nivel y variación de la equidad en la educación media de Argentina. Revista Iberoamericana de Educación, 34(4), 1-18.

Cervini, R. (2006). Los efectos de la escuela y del aula sobre el logro en matemáticas y en lengua de la educación secundaria. Un modelo multinivel. Perfiles Educativos, 27(112), 68-97.

Cervini, R. (2009). Comparando la inequidad en los logros escolares de la educación primaria y secundaria en Argentina: Un estudio multinivel. Revista Iberoamericana sobre Calidad, Eficacia y Cambio en Educación, 7(1), 5-21.

Cervini, R. (2010). El efecto escuela en la educacion primaria y secundaria: El caso de Argentina. Revista Iberoamericana sobre Calidad, Eficacia y Cambio en Educación, 8(1), 7-25.

Cervini, R. (2012). El efecto escuela en países de América Latina: Reanalizando los datos del SERCE. Archivos Analíticos de Políticas Educativas, 20(39), 1-25.

Cervini, R., Dari, N., y Quiroz, S. (2016). Las Determinaciones Socioeconómicas sobre la distribución de los Aprendizajes Escolares. Los Datos del TERCE. Revista Iberoamericana sobre Calidad, Eficacia y Cambio en Educación, 14(4), 61 -79. doi:10.15366/reice2016.14.4.003

Consejería de Educación y Ciencia del Principado de Asturias (2007). Evaluación de Diagnóstico Asturias 2007. Procesos y resultados. Oviedo: Servicio de Evaluación y Calidad. Recuperado de https://goo.gl/uZT7Ck

Cunha, J., Rosario, P., Macedo, L., Nunes, A. R., Fuentes, S., Pinto, R., y Suárez, N. (2015). Parents' conceptions of their homework involvement in elementary school. Psicothema, 27(2), 159-165. doi: 10.7334/psicothema2014.210

Doneschi, A. (2017). Desigualdad de aprendizajes en Uruguay:determinantes de los resultados de PISA 2012. (Tesis de maestría inédita), Universidad de la República, Montevideo. Recuperado de https://goo.gl/YYnmWX

Duarte, J., Bos, S., Moreno, M., y Morduchowicz, A. (2013). Equidad de los aprendizajes en Chile: Tendencias 1999-2017. Washington, D.C.: Banco Interamericano de Desarrollo. Recuperado de https://goo.gl/0pzM5F

Fernández-Alonso, R., Álvarez-Díaz, M., SuarezAlvarez, J., \& Muñiz, J. (2017). Students' achievement and homework assignment strategies. Frontiers in Psychology, 8, 286. doi:10.3389/fpsyg.2017.00286

Fernández-Alonso, R., y Muñiz, J. (2011). Diseño de cuadernillos para la evaluación de las Competencias Básicas. Aula Abierta, 39(2), 3-34.

Fernández-Alonso, R., Suárez-Álvarez, J., y Muñiz, J. (2012). Imputación de datos perdidos en las evaluaciones diagnósticas educativas. Psicothema, 24(1), 167-175.

Fernández-Alonso, R., Suárez-Álvarez, J., \& Muñiz, J. (2015). Adolescents' homework performance in mathematics and science: Personal factors and teaching practices. Journal of Educational Psychology, 107(4), 1075-1085. doi:10.1037/ edu0000032

Fernández-Alonso, R., Suárez-Álvarez, J., \& Muñiz, J. (2016). Homework and performance in mathematics: the role of the teacher, the family and the student's background. Revista de Psicodidáctica, 27(1), 5-23. doi:10.1387/ RevPsicodidact. 13939

Ferrão, M. E., y Fernandes, C. (2001). A escola brasileira faz diferença? Uma investigaçao dos efeitos da esco la na proficiência em Matemática dos alunos da $4^{a}$ série. en C. Franco (Ed.), Promoçao, ciclos e avaliaçao educacional (pp.155-172). Curitiba: Artmed.

Foces, J. A. (2015). PISA, IDE e IPE: Evidencia empírica de las desigualdades educativas entre las regiones españolas. Revista de Psicología y Educación, 10(1), 173-192.

Gobierno del Principado de Asturias. (2016). La repetición escolar: hechos y creencias. Informes de Evaluación, 2. Recuperado de https://goo.gl/ MKsGVn

Joncas, M., \& Foy, P. (2012). Sample Design in TIMSS and PIRLS. In M. O. Martin \& I. V. S. Mullis (Eds.), Methods and Procedures in TIMSS and PIRLS 2011. Chestnut Hill, MA: TIMSS and PIRLS International Study Centre, Boston College.

Martínez-Arias, R. (2010). La evaluación del desempeño. Papeles del Psicólogo, 37(1), 85-96.

Martínez-Arias, R., Gaviria-Soto, J. L., y CastroMorera, M. (2009). Concepto y evolución de los modelos de valor añadido en educación. Revista de Educación 348, 15-45.

Miralles, M. J., Castejón, L., Pérez, A., y Gilar, R. (2012). El análisis de los efectos de la escuela sobre el rendimiento académico en matemáticas: Un análisis multinivel con datos de PISA 2003. Revista de Psicología y Educación, 7(1), 83-110.

Mislevy, R. J., Beaton, A., Kaplan, B., \& Sheehan, K. (1992). Estimating population characterstics from sparse matrix samples of item responses. Journal of Education Measurement, 29(2), $131-161$. doi:10.1111/i.1745-3984.1992. tb00371.x

Murillo, F. J. (2003). La investigación sobre Eficacia Escolar en Iberoamerica. Revisión Internacional sobre el Estado del Arte. Bogotá: CAB/CIDE. 
Murillo, F. J. (2005). La investigación sobre eficacia escolar. Barcelona: Octaedro.

Murillo, F. J. (2007). School Effectiveness Research in Latin America. en T. Townsend (Ed.), International Handbook of School Effectiveness and Improvement (pp.75-92). Dordrecth: Springer.

Murillo, F. J. (2008). Enfoque, situación y desafíos de la investigación sobre eficacia escolar en Latinoamérica en Eficacia escolar y factores asociados en América Latina y el Caribe (pp. 17. 48). Santiago de Chile: OREALC-UNESCO y LLECE.

Murillo, F. J., \& Román, M. (2011). ¿̇La escuela o la cuna? Evidencias sobre su aportación al rendimiento de los estudiantes de América Latina. Estudio multinivel sobre la estimación de los efectos escolares. Revista de currículum y formación del profesorado, 15(3), 27-50.

OCDE. (2002). Conocimientos y aptitudes para la vida. Primeros resultados del Programa Internacional de Evaluación de estudiantes (PISA) 2000 de la OCDE. México: Aula XXI Santillana.

OECD. (2002). Results from PISA 2000. Reading for Change: Performance and Engagement across Countries. París: OECD Publishing.

OECD. (2004). Learning for Tomorrow's World: First Results from PISA 2003: OECD Publishing.

OECD. (2007). PISA 2006: Science Competencies for Tomorrow's World: Volume 1: Analysis. París: OECD Publishing.

OECD. (2009). PISA Data Analysis Manual SPSS $\mathbb{R}$ (Second ed.). París: OECD Publishing.

OECD. (2010). PISA 2009 Results: Overcoming Social Background - Equity in Learning Opportunities and Outcomes (Volume II) doi:10.1787/9789264091504-en

OECD. (2013). PISA 2012 Results: Excellence through Equity (Volume II): Giving Every Student the Chance to Succeed doi:/10.1787/9789264201132-en

OECD. (2016). PISA 2015 Results (Volume I): Excellence and Equity in Education PISA (Ed.) doi:/10.1787/9789264266490-en

Osorio, A. y González-Cámara, M. (2016). Testing alleged superiority of the indulgent parenting style among Spanish adolescents. Psicothema, 28(4), 414-420. doi: 10.7334/psicothema2015.314

Peña-Suárez, E., Campillo-Álvarez,Á., Santarén-Rosell, M., y Muñiz, J. (2012). El papel de los centros escolares en la adquisición de la competencia científica. Revista Iberoamericana de Psicología y Salud, 3(1), 75-87.

Peña-Suárez, E., Fernández-Alonso, R., y Muñiz, J. (2009). Estimación del valor añadido de los centros educativos. Aula Abierta, 37(1), 3-18.

Piñeros, L. J., y Rodríguez, A. (1998). Los insumos escolares en la Educación Secundaria y su efecto sobre el rendimiento académico de los estudiantes: un estudio en Colombia. Washington, D.C.: Banco Mundial.
Raudenbush, S., Bryk, A., Cheong, Y. K., Congdon, R., \& du Toit, M. (2011). HLM 7 Hierarchical Linear and Nonlinear Modeling. United States of America: SSI Scientific Software International, Inc.

Reimers, F. (2002). Distintas escuelas, diferentes oportunidades. Los retos para la igualdad de oportunidades en Latinoamérica. Madrid: La Muralla.

Robert, P. (2007). The influence of educational segregation on educational achievement (Vol. RSCAS 2007/29): European University Institute.

Rodríguez-Jiménez, O., y Murillo, F. J. (2011). Estimación del efecto escuela para Colombia. Revista Internacional de Investigación en Educación, 3(6), 299-316.

Rodríguez, S., Piñeiro, I., Regueiro, B., Estevez, I., \& Val, C. (2017). Estrategias cognitivas, etapa educativa y rendimiento académico. Revista de Psicología y Educación, 12(1), 19-34.

Scheerens, J. (2016). Educational Effectiveness and Ineffectiveness a Critical Review of the Knowledge Base: Springer Netherlands. doi: 10.1007/978-94017-7459-8 1

Scheerens, J., Witziers, B., \& Steen, R. (2013). A Meta-analysis of School Effectiveness. Revista de Educación, 361, 619-645.

Sirin, S. (2005). Socioeconomic Status and Academic Achievement: A Meta-Analytic Review of Research. Review of Educational Research, 75(3), 417-453. doi:10.3102/00346543075003417

Suárez-Álvarez, J., Fernández-Alonso, R., \& Muñiz, J. (2014). Self-concept,motivation, expectations, and socioeconomic level as predictors of academic performance inmathematics. Learning and Individual Differences, 30, 118-123. doi: 10.1016/i. lindif.2013.10.019

Teddlie, C., \& Reynolds, D. (2000). The International Handbook of School Effectiveness Research. London: Falmer Press.

Towsend, T. (2007). International handbook of school effectiveness and improvement. Dordrecth: Springer.

UNESCO-OREALC. (2016a). Recomendaciones de Políticas Educativas en América Latina en base al TERCE. Santiago de Chile: UNESCO.

UNESCO-OREALC. (2016b). Reporte Técnico Tercer Estudio Regional Comparativo y Explicativo. TERCE. Santiago de Chile: UNESCO.

UNESCO-OREALC, y LLECE. (2000). Primer estudio internacional comparativo sobre lenguaje, matemática y factores asociados, para alumnos del tercer y cuarto grado de la educación básica. Segundo Informe. Santiago de Chile. UNESCO.

UNESCO-OREALC, y LLECE. (2001). Informe Técnico. Primer estudio internacional comparativo sobre lenguaje, matemática y factores asociados, para alumnos del tercer y cuarto grado de la educación básica. Santiago de Chile: UNESCO.

UNESCO-OREALC, y LLECE. (2010). SERCE. Factores asociados al logro cognitivo de los estudiantes de América Latina y el Caribe. Santiago de Chile: UNESCO. 
UNESCO-OREALC, y LLECE. (2016a). Informe de resultados del Tercer Estudio Regional Comparativo y Explicativo. Factores Asociados. Santiago de Chile: UNESCO.

UNESCO-OREALC, \& LLECE. (2016b). Informe de resultados del Tercer Estudio Regional Comparativo y Explicativo. Logros de aprendizaje. Santiago de Chile: UNESCO.
UNESCO. (2000). Marco de Acción de Dakar. Educación para Todos cumplir nuestros compromisos comunes. Recuperado de https:// goo.gl/BEao3p

Zorrilla, M. (2009). ¿Cuál es la aportación de la escuela secundaria mexicana en el rendimiento de los alumnos en Matemáticas y Español? Revista electrónica de investigación educativa, 17(2), 1-29.

Revista de Psicología y Educación / Journal of Psychology and Education, 201 7, 12(2), 138-154 www.rpye.es Doi: https://doi.org/10.23923/rpye2017.12.152
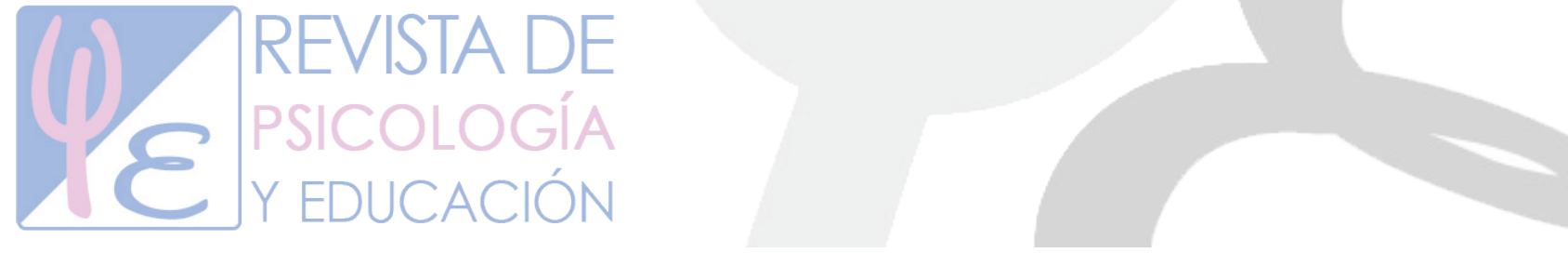